\title{
Thoracic Outlet Syndrome: Do we have Clinical Tests as Predictors For the OUtCome AFter Surgery?
}

\author{
M. Sadeghi-Azandaryani1,*, D. Bürklein ${ }^{1, *}$, A. Ozimek ${ }^{1}$, C. Geiger ${ }^{3}$, N. Mendl ${ }^{1}$, B. Steckmeier ${ }^{1}$, J. Heyn ${ }^{1,2}$ \\ ${ }^{1}$ Department of Vascular Surgery - city campus, University Hospital Munich, Munich, Germany, \\ ${ }^{2}$ Department of Anaesthesiology - Grosshadern, University Hospital Munich, Munich, Germany, \\ ${ }^{3}$ Department of Cardiology and Intensive Care Medicine, Bogenhausen Hospital, Munich, Germany
}

\begin{abstract}
Objective: Thoracic outlet syndrome (TOS) is a clinical phenomenon resulting from compression of the neurovascular structures at the superior aperture of the thorax which presents with varying symptoms. Regarding to the varying symptoms, the diagnosis of TOS seems to be a challenge and predictors for the outcome are rare. The purpose of this study was therefore to analyze the different clinical examinations and tests relative to their prediction of the clinical outcome subsequent to surgery.

Methods: During a period of five years, 56 patients were diagnosed with TOS. Medical history, clinical tests, operative procedure and complications were recorded and analysed. Mean follow-up of the patients was $55.6 \pm 45.5$ months, median age of the patients was $36.4 \pm 12.5$ years.

Results: Different clinical tests for TOS showed an acceptable sensitivity overall, but a poor specificity. A positive test was not associated with a poor outcome. Analyses of the systolic blood pressure before and after exercise showed, that a distinct decrease in blood pressure of the affected side after exercises was associated with a poor outcome $(p=0.0027)$.

Conclusions: Clinical tests for TOS show a good sensitivity, but a poor specificity and cannot be used as predictors for the outcome. A distinct decrease in blood pressure of the affected side after exercises was associated with poor outcome and might be useful to predict the patients' outcome.
\end{abstract}

Key words: Thoracic outlet syndrome, cervical rib, clinical tests, blood pressure, outcome

\section{INTRODUCTION}

Thoracic outlet syndrome (TOS) may cause several diverse clinical disorders of the upper limb, such as pain, paraesthesia and dysaesthesia. These symptoms result from compression of neuronal or vascular structures within the space delimited by the scalene muscles, the

*Mojtaba Sadeghi-Azandaryani and Dominik Bürklein contributed equally to this work. clavicle, and the first rib [1]. Compression of these neuronal and vascular structures may be secondary to anatomic bony anomalies such as cervical rib, tumour or callus formation after trauma. However, hypertrophy of the scalene muscles or the smaller pectoral muscle at the thoracic outlet can also be a causative factor for TOS $[1,2]$.

Different positional and compressive manoeuvres such as Adson's test, Eden's test, Wright's test, Greenstone test or the Elevated Arm Stress Test (EAST) can reproduce the symptoms of TOS. Doppler sonography, x-ray, electromyographs and arteriography may also show pathological results in these patients. The diagnosis of TOS remains difficult, as the different examinations are not specific for TOS. Furthermore, a variety of diseases can mimic the presentation of TOS $[3,4]$.

Physiotherapy may be the treatment of choice in patients with mild forms of TOS. Patients who do not benefit from physiotherapy should undergo surgical treatment, whereby several procedures, such as transaxillary, supraclavicular, infraclavicular and transthoracic incision, have been described. Since its introduction by Roos, the transaxillary approach has become the most popular surgical procedure in patients with TOS $[5,6]$.

From a recent review of the literature, very few reports analysing the different diagnostic options in patients with TOS were to be found; the purpose of this study was therefore to analyse the different clinical tests relative to their prediction of the clinical outcome subsequent to surgery.

\section{Patients And Methods}

During a period of five years, 56 patients (36 female and 20 male who underwent a total of 64 operations) were admitted to our vascular surgical unit and diagnosed with TOS. Afterwards, these patients were operated and followed up in a prospective manner for 55.6 \pm 45.5 months. The median age was $36.4 \pm 12.5$ years (range 17-70 years) and the majority $(64.3 \%)$ were female. Before being transferred to our outpatient clinic, these patients had been seen by an average of $3.2 \pm$ 1.1 different physicians. Mean duration of symptoms was $46.0 \pm 83.5$ months. 


\section{Diagnostic Studies}

At presentation, history was taken and physical examination was performed by three experienced consultants. Clinical examination included skin colour, swelling, weakness, sensibility of arm and hand. Additional positional and compressive manoeuvres such as Adson's test, Tinel's test, Eden's test, Wright's test, Greenstone test and the EAST test were performed. Finally, blood pressure was measured (bilaterally) before and after exercising. All patients were examined using the same protocol (patients were placed in a standing position and had to perform the EAST test, blood pressure was measured directly after exercise on the dominant upper arm using a Riva-Rocci sphygmomanometer; after a pause of ten minutes, the test was repeated on the contralateral arm).

\section{Additional Examinations}

In some cases the following additional studies were performed: upper limb angiography, electromyography, Doppler sonography and x-ray. Diagnosis of TOS was established on the basis of history, physical examination, angiography, electromyography and Doppler findings. In $60.7 \%$ of the patients TOS was diagnosed on their left side and in $39.3 \%$ on their right side.

\section{INDICATION FOR SURGERY}

Conservative treatment was attempted prior to surgery (except in patients with acute vascular forms of TOS), but proved unsuccessful in all patients. Indications for surgery were persistent symptoms of TOS after a minimum of 3 to 6 months of conservative therapy. Patients with severe symptoms, emergencies and cervical ribs underwent surgery after a shorter period to prevent further damage of vessels or nerves, and of embolism in the arms. In the event of surgery, the operation was performed by two experienced surgeons.

\section{SURGERY}

During surgery under general anaesthesia, two different procedures were performed - a transaxillary approach and a supraclavicular approach. The transaxillary approach was performed in the majority of cases $(87.5 \%)$.

\section{Data Collection And Follow-up}

The median follow up was $55.6 \pm 45.5$ months, clinical post-operative follow-up was available for all cases and included a clinical interview and examination by one of the three consultants. Patients were questioned about their amount of pain, activity status and overall satisfaction for a minimum of three months post surgery. Outcome was graded "good" when symptoms vanished and "fair" when symptoms decreased and the patients could return to their pre-illness activities. Outcome was graded as "poor" when symptoms did not improve or worsened, and the patient could not return to their pre-illness activities. Doppler sonography was performed post-operatively in all cases and electromyography was additionally performed in 36\%; these additional examinations, however, did not reveal any pathological findings.

\section{STATISTICAL ANALYSIS}

For statistical analyses of the data, SPSS 11.0 was used (SPSS Inc., Chicago, IL, USA). The chi-squared and Mann-Whitney $U$ test was used to evaluate the operative outcome, anamnestic findings, clinical examinations, operative approach and complications. A p < 0.05 was considered statistically significant. All continuous data are expressed as mean $\pm \mathrm{SD}$.

\section{RESULTS}

Interviews and examination during follow-up showed a "good" outcome in $42.2 \% .43 .8 \%$ of our patients showed a "fair" outcome and the remaining $13.0 \%$ had a "poor" outcome.

Statistical analyses of different demographic data and anamnestic findings, such as sex, age, height, weight, hypertension, trauma, tobacco use, duration of symptoms and number of medical doctors involved, did not show any association with the outcome after operation ( $p>0.05$, data not shown). The majority of our patients had neurovascular TOS $(67.2 \%)$, followed by patients with arteriovenous $(10.9 \%)$, arterial $(7.8 \%)$, neurological $(7.8 \%)$ and venous TOS (6.3\%). The character of TOS did not show any association with the outcome ( $p>0.05)$.

Sensitivity of clinical tests was acceptable overall (mean $72 \%$ ). The EAST test showed the highest sensitivity with $98 \%$, followed by the Adson (sensitivity: 92\%) and Eden tests (sensitivity: 92\%). In contrast,

Table 1. Anatomic abnormalities of patients with TOS, which could be find during operation.

\begin{tabular}{lcc}
\hline & Number & Percentage \\
\hline First Rib & 55 & $85.9 \%$ \\
Compressive fibrous structures & 26 & $40.6 \%$ \\
Cervical Rib & 16 & $25.0 \%$ \\
Scalenus muscle & 5 & $7.8 \%$ \\
Subclavian muscle & 2 & $3.1 \%$ \\
Pectoralis muscle & 2 & $3.1 \%$ \\
Clavicle fracture & 2 & $3.1 \%$ \\
\hline
\end{tabular}

Table 2. Complications after operation.

\begin{tabular}{lcc}
\hline & Number & Percentage \\
\hline Pleural effusion & 7 & $10.9 \%$ \\
Pneumothorax & 5 & $7.8 \%$ \\
Hematothorax & 4 & $6.3 \%$ \\
Bleeding & 3 & $4.7 \%$ \\
Nerve injury & 3 & $4.7 \%$ \\
Vessel injury & 2 & $3.1 \%$ \\
Wound infection & 1 & $1.6 \%$ \\
\hline
\end{tabular}




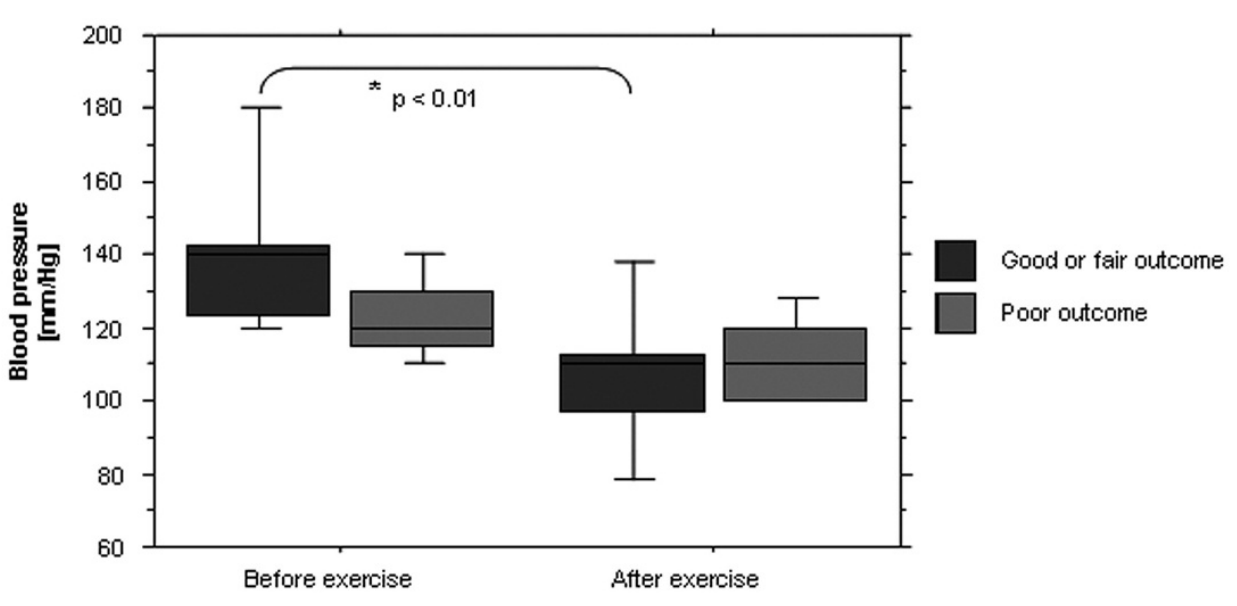

Fig. 1. Measurement of systolic blood pressure of the afflicted side before and after exercise. the sensitivity of the Hoffmann test $(47 \%)$ was low. Nevertheless, a positive EAST, Eden, Adson, Greenstone or Adson test was not associated with a poorer outcome ( $\mathrm{p} \geq 0.05)$.

Systolic blood pressure was measured before and after exercise (Fig. 1). Mean systolic blood pressure of the afflicted side in the group of patients with good or fair outcome $(85.9 \%$ of all patients) showed an average systolic blood pressure of $123.1 \pm 12.5 \mathrm{mmHg}$ before exercise and $108.9 \pm 12.8 \mathrm{mmHg}$ after exercise (average decrease: $16.2 \pm 9.6 \mathrm{mmHg}$ ). A decrease in blood pressure of more than $25 \mathrm{mmHg}$ could not be found in this group. In the group of patients with a poor outcome, the systolic blood pressure before exercise was $140.6 \pm 24.6 \mathrm{mmHg}$ and $106.7 \pm 21.8 \mathrm{mmHg}$ after exercise (average decrease: $35.0 \pm 14.1 \mathrm{mmHg}$ ). Statistical analyses showed that a distinct decrease in blood pressure after exercises was associated with a poorer outcome $(p=0.0027)$.

Surgical approaches involved two different methods (supraclavicular or transaxillary); whereby the operative approach selected was not seen to be associated with a poorer outcome ( $\mathrm{p} \geq 0.05$ ). Intra-operatively, different abnormalities were observed, which are summarized in Table 1. Several complications occurred during surgery and post-operatively. These complications are listed in Table 2. Statistic analyses showed no association of pathologies or peri-operative complications and a poor outcome.

\section{Discussion}

Thoracic outlet syndrome is a clinical phenomenon resulting from compression of the neurovascular structures at the superior aperture of the thorax which presents with varying symptoms. These symptoms, which appear as a consequence of anatomic variations, were first described by Coote in 1861 [7] and the term TOS was used in the literature for the first time by Peet and Rob in the late 1950ies [8, 9].

To date, the diagnosis of TOS seems to be a challenge for a lot of physicians. In the current study, our patients were seen by an average of $3.2 \pm 1.1$ physicians before the diagnosis was established; a good explanation possibly being that patients with TOS present a broad spectrum of symptoms. Therefore, a lot of differential diagnoses are possible; fortunately, the length of symptoms and the number of consulted physicians was not associated with a poorer outcome.

The aetiology of TOS is multiple. Trauma has been implicated in a number of cases. It is necessary to distinguish between minor and major trauma; minor trauma without bone or neurovascular lesions causes most cases of TOS as a result of remodelling of muscle fibres during healing $[10,11]$. Therefore, it is not unexpected that anatomic abnormalities can frequently be found in patients with TOS. These anomalies include cervical rib, first rib or soft tissue structures [12-14]. In our study, 26 patients presented compressive fibrous structures as reasons for TOS. Trauma of the clavicle or muscle hypertrophy could also be seen in some cases (Table 1). However, the type of abnormality was not associated with a poorer outcome.

Different diagnostic tests (e.g. Adson, Greenstone, EAST, Wright or Eden) have been suggested as diagnostic tools in patients with TOS. The sensitivity of these tests is acceptable, as shown in our study, although a disadvantage to these tests might be their specificity, which is considered to be poor. Apart from Wright's test (specificity: 90\%), the specificity of these tests is less than $80 \%$ and the different tests failed to be a predictor for a poor outcome [13].

Measurement of the blood pressure is a simple clinical test, which is also cheap. As this study shows, a distinct decrease in systolic blood pressure of the affected side after exercises was a negative predictor for the outcome of patients with TOS after surgery. Therefore, this test might be useful to predict the patients' outcome.

A poorer outcome was not associated with the aetiology of TOS, the operative technique, the length of the operation, a cervical rib or pathological findings during electrophysiological or neurological examination. Especially pathological findings during electrophysiological or neurological examination were not associated with a distinct decrease in blood pressure on the affected side after exercises. An explanation of these findings could be that a distinct decrease of blood pressure could be an indirect sign of an excessive compression of vessels and neural structures. This compression might lead to a beginning damage or irritation of the neural structures, which is not detectable by electrophysiological or neurological examination. Therefore, this easy practical test might also be 
useful as an indirect indicator for the grade of neural and vascular compression and as an indirect sign for beginning damage and irritation. In these patients, an earlier operative therapy (instead of conservative therapy) might be useful. Further randomized, prospective studies are necessary to verify this hypothesis.

However, conservative therapy should usually be attempted prior to surgery. In patients with persistent symptoms of TOS for more than 6 months of conservative therapy, surgery should be considered. There are different objectives of surgical treatment for neurogenic and vascular TOS. For neurogenic TOS, the objective is to diminish the symptoms, while in vascular TOS the operative indication is the prevention of thrombosis or weakness of the arm during exercises. Surgical decompression of the thoracic outlet usually leads to significant benefit in patients with TOS $[5,15$, 16]. Since its introduction by Roos, the transaxillary approach has become the most popular surgical procedure in patients with TOS [17]. In general, the transaxillary approach provides a good exposure for the resection of cervical ribs, the first rib and excision of fibrous ligaments and the scalenus muscle associated with perfect cosmetic results [18]. Most of our patients were operated using a transaxillary approach because of the different advantages mentioned above. Regarding a poor outcome, we could not find a disadvantage to any of the different surgical procedures.

Finally, our study has two limitations. First of all, we only included patients which were diagnosed with TOS. Therefore, a control group of patients without TOS is lacking. A control group would be helpful to evaluate the specificity of the different clinical tests used in this study. However, there is one study that provides these data for some tests, which were used in our case [13]. Secondly, due to our surgical unit (vascular centre), the majority of patients showed a vascular component of TOS $(95.0 \%)$. Therefore, the statements made in this study are mainly valid for patients with vascular TOS.

\section{CONCLUSION}

TOS is a disease with a broad spectrum of symptoms, which leads to a delayed diagnosis. Clinical tests for TOS show a good sensitivity, but a poor specificity and cannot be used as predictors for the outcome. Measurement of the blood pressure is a simple clinical test and a distinct decrease in blood pressure of the affected side after exercises was associated with a poorer outcome. Therefore, this test might be useful to predict the patients' outcome.

\section{REFERENCES}

1. Urschel HC Jr, Razzuk MA. Neurovascular compression in the thoracic outlet: changing management over 50 years. Ann Surg 1998; 228:609-617.

2. Redenbach DM, Nelems B. A comparative study of structures comprising the thoracic outlet in 250 human cadavers and 72 surgical cases of thoracic outlet syndrome. Eur J Cardiothorac Sur 1998; 13:353-360.
3. Huang JH, Zager EL. Thoracic outlet syndrome. Neurosurgery 2004; 55:897-903.

4. Novak CB, Mackinnon SE, Patterson GA. Evaluation of patients with thoracic outlet syndrome. Hand Surg 1993; 18:292-299 .

5. Roos DB. New concepts of thoracic outlet syndrome that explain etiology, symptoms, diagnosis and treatment. J Vasc Surg 1079; 13:313-321.

6. Lindgren K. Conservative treatment of thoracic outlet syndrome: a 2-year follow-up Arch Phys Med Rehabil 1997; 78:373-378.

7. Coote H. Exostosis of the left transverse process of the seventh cervical vertebrae, suurounded by blood vessels and nerves, successful removel. Lancet 1861; 1:360-361.

8. Rob CG, Standeven A. Arterial occlusion complicating thoracic outlet compression syndrome. Brit Med J 1958; 20:709-712.

9. Peet RM, Henriksen JD, Anderson TP, Martin GM. Thoracic outlet syndrome: Evaluation of a therapeutic exercise program. Proc Staff Meet Mayo Clin 1956; 31:281287.

10. Sanders RJ, Jackson CG, Banchero N, Pearce WH. Scalene muscle abnormalities in traumatic thoracic outlet syndrome. Am J Surg 1990; 159:231-236.

11. Ellison DW, Wood VE. Trauma related thoracic outlet syndrome. J Hand Surg 1994; 19:424-426.

12. Sanders RJ, Hammond SL. Management of cervical ribs and anomalous first ribs causing neurogenic thoracic outletsyndrome. J Vasc Surg 2002; 36:51-56.

13. Gillard J, Pérez-Cousin M, Hachulla E, Remy J, Hurtevent JF, Vinckier L, Thévenon A, Duquesnoy B. Diagnosing thoracic outlet syndrome: contribution of provocative tests, ultrasonography, electrophysiology, and helical computed tomography in 48 patients. Joint Bone Spine 2001; 68:416-424.

14. Davidovic LB, Kostic DM, Jakovljevic NS, Kuzmanovic IL, Simic TM. Vascular thoracic outlet syndrome. World J Surg 2003; 27:545-550.

15. Cordobes-Gual J, Lozano-Vilardell P, Torreguitart-Mirada N, Lara-Hernandez R, Riera-Vazquez R, Julia-Montoya J. Prospective study of the functional recovery after surgery for thoracic outlet syndrome. Eur J Vasc Endovasc Surg 2008; 35:79-83.

16. Athanassiadi K, Kalavrouziotis G, Karydakis K, Bellenis I. Treatment of thoracic outlet syndrome: long-term results. World J Surg 2001; 25:553-557.

17. Sheth RN, Campbell JN. Surgical treatment of thoracic outlet syndrome: a randomized trial comparing two operations. J Neurosurg Spine 2005; 3:355-363.

18. Han S, Yildirim E, Dural K, Ozisik K, Yazkan R, Sakinci U. Transaxillary approach in thoracic outlet syndrome: the importance of resection of the first-rib. European J Cardio-thoracic Surg 2003; 24:428-433.

Received: February 12, 2009 / Accepted: June 4, 2009

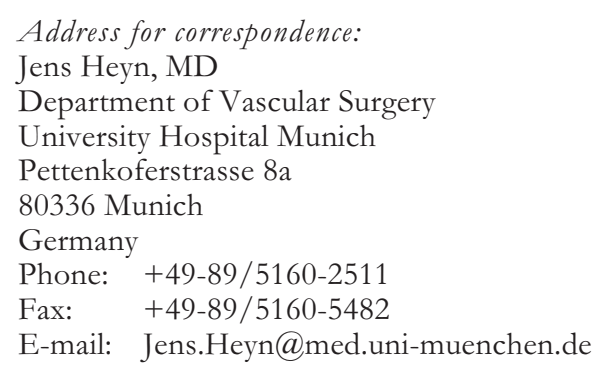

